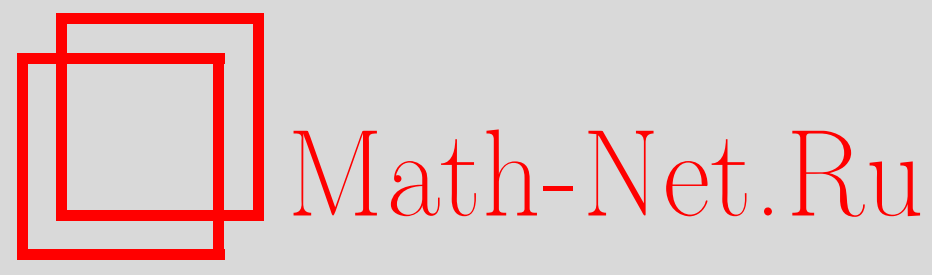

М. Л. Буряков, О связи уровня аффинности с криптографическими параметрами булевых функций, Дискрет. матем., 2008, том 20, выпуск 2, 3-14

DOI: https://doi.org/10.4213/dm999

Использование Общероссийского математического портала Math-Net.Ru подразумевает, что вы прочитали и согласны с пользовательским соглашением http://www . mathnet.ru/rus/agreement

Параметры загрузки:

IP: 52.205 .19 .152

26 апреля 2023 г., 12:30:20 
УДК 519.7

\title{
О связи уровня аффинности с криптографическими параметрами булевых функций
}

\author{
() 2008 г. М. Л. Буряков
}

\begin{abstract}
В работе рассматриваются соотношения, связывающие значение уровня аффинности булевых функций и другие криптографические параметры булевых функций, такие как нелинейность, порядок корреляционной и алгебраической иммунности и другие. Для определенного класса функций доказывается $N P$-трудность задачи определения уровня аффинности.

Работа выполнена при поддержке Российского фонда фундаментальных исследований, проект 07-01-00154.
\end{abstract}

\section{1. Введение}

Система булевых уравнений является одной из наиболее часто используемых математических моделей при анализе криптографических конструкций. В частности, с помощью систем булевых уравнений описывается функционирование комбинирующих и фильтрующих генераторов псевдослучайных последовательностей (см. [7]). Кроме того, необходимость изучения и решения систем булевых уравнений возникает в ряде задач теории конечных автоматов, теории кодирования и других.

Одним из эффективных классов методов решения систем булевых уравнений является класс методов, связанных с линеаризацией исходной системы уравнений. Описание различных методов линеаризации (в том числе с введением новых переменных и уравнений) можно найти, например, в работах $[9,10,11,12]$.

В [1] был предложен метод линеаризации исходной системы булевых уравнений без введения новых переменных. Параметром, характеризующим эффективность этого метода, является уровень аффинности булевых функций, введенный в [2].

При выборе той или иной функции в качестве элемента криптографических систем обычно учитывают многие параметры этой функции: уравновешенность, алгебраическую степень, нелинейность, уровень корреляционной иммунности, уровень алгебраической иммунности и другие. При этом часто стремятся одновременно улучшить все эти показатели. К сожалению, результаты показывают, что при улучшении одного свойства булевой функции, часто ухудшаются другие ее свойства, так как большинство из приведенных выше параметров находятся в жестких связях.

Настоящая работа посвящена описанию подобных связей по отношению к уровню аффинности булевых функций. Найдены соотношения, связывающие уровень аффинности с практически всеми основными криптографическими параметрами. 


\section{2. Основные обозначения и определения}

Введем некоторые обозначения и напомним необходимые свойства булевых функций (см., например, [4]).

Будем обозначать $F_{2}$ конечное поле из двух элементов, $V_{n}=F_{2}^{n}$ линейное пространство вектор-столбцов размерности $n$ над полем $F_{2}$. Вес Хэмминга двоичного вектора $\mathbf{x}=\left(x_{1}, \ldots, x_{n}\right)$ определяется как

$$
\mathrm{wt}(\mathbf{x})=\sum_{i=1}^{n} x_{i}
$$

Обозначим $\mathscr{F}_{n}$ множество булевых функций от $n$ переменных, то есть множество всех возможных отображений из $V_{n}$ в $F_{2}$. Любая функция из $\mathscr{F}_{n}$ может быть представлена в виде полинома, называемого алгебраической нормальной формой (АНФ) этой функции,

$$
f\left(x_{1}, \ldots, x_{n}\right)=\bigoplus_{\mathbf{u} \in V_{n}} \lambda_{\mathbf{u}} \prod_{i=1}^{n} x_{i}^{u_{i}}, \quad \lambda_{\mathbf{u}} \in F_{2},
$$

при этом коэффициенты $\lambda_{\mathbf{u}}$ выражаются через значения функции следующим образом:

$$
\lambda_{\mathbf{u}}=\bigoplus_{\mathbf{x} \preccurlyeq \mathbf{u}} f(\mathbf{x}),
$$

где $\left(x_{1}, \ldots, x_{n}\right) \preccurlyeq\left(u_{1}, \ldots, u_{n}\right)$ тогда и только тогда, когда $x_{i} \leqslant u_{i}$ для всех $1 \leqslant i \leqslant n$. Алгебраической степенью функции $f$, обозначаемой $\operatorname{deg}(f)$, является максимальное значение $\operatorname{wt}(\mathbf{u})$, для которого $\lambda_{\mathbf{u}} \neq 0$. Если $\operatorname{deg}(f) \leqslant 1$, то такая функция называется аффинной. Множество всех аффинных функций от $n$ переменных обозначим через $\mathscr{A}_{n}$. Заметим также, что любая аффинная функция $f \in \mathscr{A}_{n}$ имеет вид $f(\mathbf{x})=\langle\mathbf{x}, \mathbf{a}\rangle \oplus \varepsilon$, где

$$
\langle\mathbf{x}, \mathbf{a}\rangle=\bigoplus_{i=1}^{n} x_{i} a_{i}
$$

- скалярное произведение векторов из $V_{n}, \varepsilon \in F_{2}$. Для подобного представления аффинных функций введем обозначение $f(\mathbf{x})=\ell_{\mathbf{a}, \varepsilon}(\mathbf{x})$.

Любая функция $f(\mathbf{x}) \in \mathscr{F}_{n}$ может быть представлена в виде двоичного вектора длины $2^{n}$, состоящего из всех значений этой функции при $\mathbf{x} \in V_{n}$ (при этом порядок элементов $V_{n}$ считаем фиксированным). Будем называть весом функции вес соответствующего ей вектора и обозначать этот вес $\operatorname{wt}(f)$. Булева функция $f \in \mathscr{F}_{n}$ называется уравновешенной, если $\operatorname{wt}(f)=2^{n-1}$.

Пусть $f \in \mathscr{F}_{n}$. Для наборов $1 \leqslant i_{1}<\ldots<i_{k} \leqslant n, \mathbf{b}=\left(b_{1}, b_{2}, \ldots, b_{k}\right)^{\top} \in V_{k}$ при $k \leqslant n$ обозначим через $f_{i_{1}, \ldots, i_{k}}^{b_{1}, \ldots, b_{k}}$ булеву функцию из $\mathscr{F}_{n-k}$, полученную из $f$ фиксацией переменных $x_{i_{1}}=b_{1}, \ldots, x_{i_{k}}=b_{k}$ и называемую подфункцией функции $f$.

Для произвольной функции $f \in \mathscr{F}_{n}$ и произвольного $\mathbf{u} \in V_{n}$ преобразование УолшаАдамара функции $f$ определяется выражением

$$
\mathrm{W}_{f}(\mathbf{u})=\sum_{\mathbf{x} \in V_{n}}(-1)^{f(\mathbf{x}) \oplus\langle\mathbf{u}, \mathbf{x}\rangle} .
$$

Набор целых чисел $\left\{\mathrm{W}_{f}(\mathbf{u}) \mid \mathbf{u} \in V_{n}\right\}$ называется спектром функции $f$, а каждое число $\mathrm{W}_{f}(\mathbf{u})$ спектральным коэффициентом Уолша-Адамара функции $f$ (см., например, [4]). 
Нелинейность $\mathrm{N}_{f}$ булевой функции $f \in \mathscr{F}_{n}$ определяется как расстояние Хэмминга между функцией $f$ и множеством $\mathscr{A}_{n}$. Это значение может быть вычислено, исходя из спектра функции $f$, по формуле

$$
\mathrm{N}_{f}=2^{n-1}-\frac{1}{2} \max _{\mathbf{u} \in V_{n}}\left|\mathrm{~W}_{f}(\mathbf{u})\right| .
$$

Высокое значение нелинейности является важным криптографическим условием, накладываемым на используемые на практике булевы функции, так как это позволяет в ряде случаев избежать хорошей линейной аппроксимации этих функций (см., например, [13]).

Для случая четного $n=2 k$ известно, что максимальное значение нелинейности достигается для функций, спектр которых состоит из двух значений коэффициентов Уолша-Адамара $\pm 2^{k}$. Такие функции называются максимально-нелинейными или бентфункциями (см. [14]). В дальнейшем мы будем использовать термин бент-функция. Обозначим множество всех бент-функций от $n=2 k$ переменных через $\mathscr{B}_{n}$.

Кроме бент-функций, рассматриваются так называемые платовидные функции, спектр которых состоит из трех возможных значений $\left\{0, \pm 2^{n-r}\right\}$, здесь $n-$ число переменных функции, $r-$ некоторое целое число, $0 \leqslant r \leqslant n$. В этом случаем число $2 r$ называют порядком платовидности. Можно считать бент-функции из $\mathscr{B}_{2 k}$ платовидными функциями порядка $k$.

Функция $f \in \mathscr{F}_{n}$ называется корреляционно-иммунной порядка $m, 1 \leqslant m \leqslant n$, если для любых наборов $1 \leqslant i_{1}<i_{2}<\ldots<i_{m} \leqslant n, a_{1}, \ldots, a_{m} \in F_{2}$ выполняется соотношение

$$
\operatorname{wt}\left(f_{i_{1}, \ldots, i_{m}}^{a_{1}, \ldots, a_{m}}\right)=\frac{\operatorname{wt}(f)}{2^{m}} .
$$

Уравновешенная корреляционно-иммунная порядка $m$ функция называется $m$-устойчивой.

Корреляционная иммунность отражает способность функции противостоять корреляционному анализу потоковых шифров (например, построенных на основе комбинирующих или фильтрующих генераторов).

Естественным образом введем следующие обозначения: если $f \in \mathscr{F}_{n}$, то

$$
\operatorname{cor}(f)=\max \{m \in \mathbf{N} \mid f \text { корреляционно-иммунна порядка } m\},
$$

если $f \in \mathscr{F}_{n}$ и $\operatorname{wt}(f)=2^{n-1}$, то

$$
\operatorname{sut}(f)=\max \{m \in \mathbf{N} \mid f \text { является } m \text {-устойчивой }\} \text {. }
$$

Приведем одно важное неравенство, неравенство Зигенталера [8], связывающее алгебраическую степень и порядок корреляционной иммунности функции.

Теорема 1 ([8]). Для любой $f \in \mathscr{F}_{n}$ справедливо неравенство

$$
\operatorname{deg}(f)+\operatorname{cor}(f) \leqslant n .
$$

Если, кроме того, $\operatorname{wt}(f)=2^{n-1}$, то

$$
\operatorname{deg}(f)+\operatorname{sut}(f) \leqslant n-1 .
$$

Следующие определения используются при алгебраическом методе криптоанализа и характеризуют способность функции противостоять ему (см. [12]).

Пусть $f \in \mathscr{F}_{n}$. Функция $g \in \mathscr{F}_{n}$ называется аннигилятором функции $f$, если $f g=0$. 
Порядком алгебраической иммунности для булевой функции $f \in \mathscr{F}_{n}$ называется число

$$
\mathrm{AI}(f)=\min \left\{\operatorname{deg}(g) \mid g \in \mathscr{F}_{n}, g \neq 0, f g=0 \vee(1 \oplus f) g=0\right\} .
$$

Известно (см. [15]), что для любой функции $f \in \mathscr{F}_{n}$ выполняется соотношение

$$
\mathrm{AI}(f) \leqslant\lceil n / 2\rceil
$$

В данной статье все вышеперечисленные криптографические параметры булевых функций рассматриваются в соотношениях с еще одним введенным в [2] параметром уровнем аффинности. Приведем определения.

Булева функция $f$ из $\mathscr{F}_{n}$ называется $k$-аффинной, $0 \leqslant k \leqslant n-1$, если существуют наборы $1 \leqslant i_{1}<\ldots<i_{k} \leqslant n, \mathbf{b}=\left(b_{1}, \ldots, b_{k}\right)^{\top} \in V_{k}$ такие, что $f_{i_{1}, \ldots, i_{k}}^{b_{1}, \ldots, b_{k}}$ является аффинной, то есть $\operatorname{deg} f_{i_{1}, \ldots, i_{k}}^{b_{1}, \ldots, b_{k}} \leqslant 1$.

Любая аффинная функция является 0 -аффинной, а любая функция из $\mathscr{F}_{n}$ является $(n-1)$-аффинной.

Уровнем аффинности la $(f)$ булевой функции $f$ из $\mathscr{F}_{n}$ называется минимальное неотрицательное целое число $k$, для которого $f$ является $k$-аффинной.

Так как $\mathrm{la}(f) \leqslant n-1$ для любой функции $f \in \mathscr{F}_{n}$, представляет определенный интерес класс функций, на которых достигается максимальный уровень аффинности. Этот класс описывается следующим утверждением.

Теорема 2 ([3]). Для булевой функции $f$ из $\mathscr{F}_{n}$ соотношение

$$
\operatorname{la}(f)=n-1
$$

выполнено тогда и только тогда, когда

$$
f(\mathbf{x})=\bigoplus_{i<j} x_{i} x_{j} \oplus \ell_{\mathbf{a}, \varepsilon}(\mathbf{x})
$$

для произвольных $\mathbf{a} \in V_{n}, \varepsilon \in F_{2}$.

В некоторых случаях будем использовать следующее понятие: булева функция $f$ из $\mathscr{F}_{n}$ называется сильно $k$-аффинной, $0 \leqslant k \leqslant n-1$, если существует набор $1 \leqslant i_{1}<\ldots<i_{k} \leqslant n$ такой, что для любого $\mathbf{b}=\left(b_{1}, \ldots, b_{k}\right)^{\top} \in V_{k}$ подфункция $f_{i_{1}, \ldots, i_{k}}^{b_{1}, \ldots, b_{k}}$ является аффинной.

\section{3. Уровень аффинности и нелинейность}

Следующая теорема играет основную роль в дальнейших рассуждениях.

Теорема 3. Пусть $f \in \mathscr{F}_{n}$. Тогда для коэффищиентов Уолиа-Адамара функции $f$ вььполняется неравенство

$$
\max _{\mathbf{u} \in V_{n}}\left|\mathrm{~W}_{f}(\mathbf{u})\right| \geqslant 2^{n-\mathrm{la}(f)} .
$$


Доказательство. Если $\mathrm{la}(f)=k$, то найдутся такие наборы $1 \leqslant i_{1}<\ldots<i_{k} \leqslant n$, $c_{1}, \ldots, c_{k} \in F_{2}, \mathbf{v} \in V_{n-k}$ и $\varepsilon \in F_{2}$, для которых $f_{i_{1}, \ldots, i_{k}}^{c_{1}, \ldots, c_{k}}(\mathbf{x})=\ell_{\mathbf{v}, \varepsilon}=\langle\mathbf{v}, \mathbf{x}\rangle \oplus \varepsilon$. На с. 79 в [4] доказана справедливость равенства

$$
2^{\operatorname{dim} L-n}(-1)^{\langle\mathbf{a}, \mathbf{b}\rangle} \sum_{\mathbf{x} \in L^{\perp} \oplus \mathbf{b}} \mathrm{W}_{f}(\mathbf{x})(-1)^{\langle\mathbf{a}, \mathbf{x}\rangle}=\sum_{\mathbf{x} \in \mathbf{a} \oplus L}(-1)^{f(\mathbf{x}) \oplus\langle\mathbf{b}, \mathbf{x}\rangle}
$$

для любой функции $f \in \mathscr{F}_{n}$, произвольного подпространства $L \subseteq V_{n}$ и произвольных векторов $\mathbf{a}, \mathbf{b} \in V_{n}$.

Пусть в (6) $L=\left\{\mathbf{u} \in V_{n}: u_{i_{1}}=\ldots=u_{i_{k}}=0\right\}-$ подпространство размерности $\operatorname{dim} L=n-k$ пространства $V_{n}$, вектор а такой, что его координаты $a_{j}=c_{j}$ для $j \in\left\{i_{1}, \ldots, i_{k}\right\}$, и $a_{j}=0$ для $j \notin\left\{i_{1}, \ldots, i_{k}\right\}$, и вектор $\mathbf{b}=\mathbf{v}$. Правая часть равенства (6) в этом случае есть

$$
\begin{aligned}
\sum_{\mathbf{x} \in L \oplus \mathbf{a}}(-1)^{f(\mathbf{x}) \oplus\langle\mathbf{b}, \mathbf{x}\rangle} & =\sum_{\mathbf{x} \in V_{n-k}}(-1)^{f_{i_{1}, \ldots, i_{k}}^{c_{1}, \ldots, c_{k}}(\mathbf{x}) \oplus\langle\mathbf{v}, \mathbf{x}\rangle} \\
& =\sum_{\mathbf{x} \in V_{n-k}}(-1)^{\langle\mathbf{v}, \mathbf{x}\rangle \oplus \varepsilon \oplus\langle\mathbf{v}, \mathbf{x}\rangle}=\sum_{\mathbf{x} \in V_{n-k}}(-1)^{\varepsilon}=2^{n-k}(-1)^{\varepsilon} .
\end{aligned}
$$

Подставляя это выражение в (6) и переходя к абсолютным значениям, получаем равенство

$$
\left|\sum_{\mathbf{x} \in L^{\perp} \oplus \mathbf{b}} \mathrm{W}_{f}(\mathbf{x})(-1)^{\langle\mathbf{a}, \mathbf{x}\rangle}\right|=2^{n} .
$$

Число слагаемых в левой части (7) равно $2^{n-\operatorname{dim} L}=2^{k}$, откуда непосредственно следует, что

а значит, и утверждение теоремы.

$$
\max _{\mathbf{u} \in L^{\perp} \oplus \mathbf{b}}\left|\mathrm{W}_{f}(\mathbf{u})\right| \geqslant \frac{2^{n}}{2^{k}}=2^{n-k},
$$

Теорема 3 позволяет получить связь между нелинейностью функции и уровнем аффинности, а также оценить уровень аффинности некоторых классов функций.

Следствие 1. Для любой функциии $f \in \mathscr{F}_{n}$

$$
\mathrm{N}_{f} \leqslant 2^{n-1}-2^{n-\mathrm{la}(f)-1} .
$$

Доказательство. Утверждение непосредственно следует из теоремы 3 и соотношения (1).

Следствие 2. Для любой бент-функциии $f \in \mathscr{B}_{2 k}$

$$
\mathrm{la}(f) \geqslant k \text {. }
$$

Доказательство. Утверждение следует из (8) с учетом того, что нелинейность бентфункции $f$ равна $\mathrm{N}_{f}=2^{2 k-1}-2^{k-1}$.

Следствие 3. Для любой платовидной порядка $2 r$ функции $f \in \mathscr{F}_{n}$

$$
\mathrm{la}(f) \geqslant r .
$$

Доказательство. Утверждение следует из (8) с учетом того, что нелинейность функции $f$ равна $\mathrm{N}_{f}=2^{n-1}-2^{n-r-1}$.

Заметим, что функции вида (4) являются бент-функциями для четных $n$ и платовидными при нечетных $n$, при этом уровень аффинности для них имеет максимально возможное значение $n-1$. 


\section{4. Уровень аффинности и корреляционная иммунность}

Теорема 4. Пусть $f \in \mathscr{F}_{n}, \operatorname{wt}(f) \neq 0,2^{n}, 2^{n-1}$. Тогда $\operatorname{la}(f)>\operatorname{cor}(f)$.

Доказательство. Пусть $\operatorname{cor}(f)=k$, тогда согласно (2) для любой подфункции выполняется соотношение $\operatorname{wt}\left(f_{i_{1}, \ldots, i_{k}}^{a_{1}, \ldots, a_{k}}\right)=\operatorname{wt}(f) / 2^{k}$. С другой стороны, если какая-либо из этих подфункций является аффинной, то ее вес должен быть равен одному из чисел $0,2^{n-k}, 2^{n-k-1}$, а согласно условию теоремы это не так. Таким образом, функция $f$ не является $k$-аффинной. Очевидно, что при этом она не может быть $k^{\prime}$-аффинной, где $k^{\prime}<k$, откуда следует, что $\mathrm{la}(f)>k$.

Как можно видеть, теорема 4 не затрагивает $m$-устойчивые функции, которые по определению являются уравновешенными. Для подобных функций ситуация выглядит несколько иначе, что видно из следующих примеров.

Рассмотрим функцию из $\mathscr{F}_{n}, n \geqslant 3$,

$$
f(\mathbf{x})=x_{1} x_{2} \oplus \bigoplus_{i=3}^{n} x_{i} .
$$

Можно показать (см. [4]), что эта функция является $(n-3)$-устойчивой для любого $n$. С другой стороны, очевидно, что $\mathrm{la}(f)=1$ для любого $n$, то есть для этой функции $\operatorname{la}(f)>\operatorname{sut}(f)$ при $n=3, \operatorname{la}(f)=\operatorname{sut}(f)$ при $n=4, \operatorname{la}(f)<\operatorname{sut}(f)$ при $n>4$.

Приведем еще один пример, используя следующее утверждение.

Теорема 5 ([4]). Пусть $f-$ квадратичная т-устойчивая функция из $\mathscr{F}_{n}, n \geqslant 3$, $\operatorname{deg}\left(f, x_{i}\right)=2$ для всех $i=1,2, \ldots, n$. Тогда $m \leqslant\lfloor n / 2\rfloor-1$.

Далее рассмотрим функции

$$
g_{n}(\mathbf{x})=\bigoplus_{1 \leqslant i<j \leqslant n} x_{i} x_{j}, \quad n=4 k-1, \quad k \geqslant 1 .
$$

Эти функции имеют вид (4), а значит, $\mathrm{la}\left(g_{n}\right)=n-1$. Кроме того, несложно показать, что эти функции являются уравновешенными. Поэтому из теоремы 5 следует, что $\operatorname{sut}\left(g_{n}\right)<\mathrm{la}\left(g_{n}\right)$ для любой функции $g_{n}$.

В заключение рассмотрим еще один класс функций, которые одновременно имеют высокий уровень корреляционной иммунности и высокую нелинейность. Построение таких функций представляет определенный интерес, и этой проблеме посвящено много работ (см. [5]). Будем рассматривать только случай уравновешенных функций, так как именно они ценны для криптографических приложений.

Известно соотношение, связывающее нелинейность функции и ее порядок корреляционной иммунности (см., например, [5, 4]): если $f \in \mathscr{F}_{n}, \operatorname{sut}(f)=m \leqslant n-2$, то

$$
\mathrm{N}_{f} \leqslant 2^{n-1}-2^{m+1} \text {. }
$$

Заметим, что неравенство (9) достижимо, причем только для тех функций, для которых достижимо неравенство Зигенталера (3). Исходя из (8) несложно видеть, что для подобных функций имеет место следующее утверждение.

Теорема 6. Пусть $f \in \mathscr{F}_{n}$, $\operatorname{sut}(f)=m \leqslant n-2, \mathrm{~N}_{f}=2^{n-1}-2^{m+1}$. Тогда

$$
\mathrm{la}(f) \geqslant n-m-2 .
$$




\section{5. Уровень аффинности и алгебраическая иммунность}

Пусть $S \subseteq V_{n}-$ подмножество пространства $V_{n}, f \in \mathscr{F}_{n}$. Назовем функцию $g \in \mathscr{F}_{n}$ аннигилятором функции $f$ на множестве $S$, если $g(\mathbf{x}) f(\mathbf{x})=0$ для всех $\mathbf{x} \in S$. Обозначим через $\left.\mathrm{AI}\right|_{L \oplus \mathbf{a}}(f)$ минимальную степень аннигилятора функции $f$ или $f \oplus 1$ на смежном классе $L \oplus \mathbf{a}$, при этом потребуем, чтобы функция $g$ не являлась константой 0 на этом смежном классе:

$$
\begin{aligned}
\left.\mathrm{AI}\right|_{L \oplus \mathbf{a}}(f)=\min \left\{\operatorname{deg}(g) \mid g \in \mathscr{F}_{n}: f(\mathbf{x}) g(\mathbf{x})=0\right. & \vee(1 \oplus f(\mathbf{x})) g(\mathbf{x})=0 \\
& \forall \mathbf{x} \in L \oplus \mathbf{a}, \operatorname{supp}(g) \cap L \oplus \mathbf{a} \neq \varnothing\},
\end{aligned}
$$

здесь $\operatorname{supp}(g)=\left\{\mathbf{x} \in V_{n} \mid g(\mathbf{x})=1\right\}$.

Теорема 7. Пусть $f \in \mathscr{F}_{n}, L \subseteq V_{n}-$ линейное подпространство $V_{n}$, a $\in V_{n}$. Тогда

$$
\operatorname{AI}(f) \leqslant\left.\operatorname{AI}\right|_{L \oplus \mathbf{a}}(f)+n-\operatorname{dim} L
$$

Доказательство. Пусть уровень $\left.\mathrm{AI}\right|_{L \oplus \mathbf{a}}(f)$ достигается на функции $g$. Заметим, что для дальнейших рассуждений неважно, является $g$ аннигилятором функции $f$ или $f \oplus 1$. Таким образом, $\operatorname{deg}(g)=\left.\mathrm{AI}\right|_{L \oplus \mathbf{a}}(f)$.

Рассмотрим также функцию $h(\mathbf{x}) \in \mathscr{F}_{n}$, являющуюся индикатором плоскости $L \oplus \mathbf{a}$, то есть

$$
h(\mathbf{x})=I_{L \oplus \mathbf{a}}= \begin{cases}1, & \text { если } \mathbf{x} \in L \oplus \mathbf{a}, \\ 0, & \text { если } \mathbf{x} \notin L \oplus \mathbf{a} .\end{cases}
$$

В [6] показано, что функция $h$ является кодовым словом минимального веса для кода $\mathrm{RM}(n, n-\operatorname{dim} L)$, откуда следует, что $\operatorname{deg}(h) \leqslant n-\operatorname{dim} L$.

Нетрудно видеть, что функция $g h$ является аннигилятором функции $f$ (или $f \oplus 1$ ) на всем пространстве $V_{n}$, так как для плоскости $L \oplus$ а по условию справедливо равенство $g f=0(g(f \oplus 1)=0)$, а вне этой плоскости функция $h$ равна 0 . Таким образом, $g h f=0$ $(g h(f \oplus 1)=0)$. При этом $g h \neq 0$, поскольку $g \neq 0$ на $L \oplus \mathbf{a}$. Окончательно получаем, что $\operatorname{AI}(f) \leqslant \operatorname{deg}(g h) \leqslant\left.\operatorname{AI}\right|_{L \oplus \mathbf{a}}(f)+n-\operatorname{dim} L$.

Следствие 4. Для любой функции $f \in \mathscr{F}_{n}$

$$
\operatorname{AI}(f) \leqslant \min _{L \oplus \mathbf{a}, \operatorname{dim} L \geqslant\lfloor n / 2\rfloor}\left\{\left.\mathrm{AI}\right|_{L \oplus \mathbf{a}}(f)+n-\operatorname{dim} L\right\},
$$

где минимум берется по всем плоскостям $L \oplus$ а соответствующей размерности из пространства $V_{n}$.

Доказательство. Справедливость утверждения следует из теоремы 7 в силу произвольности выбора плоскости $L \oplus \mathbf{a}$ и того факта, что для любой функции $f \in \mathscr{F}_{n}$ выполняется соотношение $\mathrm{AI}(f) \leqslant\lceil n / 2\rceil$.

Следствие 5. Для любой функщии $f \in \mathscr{F}_{n}$

$$
\mathrm{AI}(f) \leqslant \min _{0 \leqslant k \leqslant\lceil n / 2\rceil, 0 \leqslant i_{1}<\ldots<i_{k} \leqslant n, \mathbf{c} \in V_{k}}\left\{\operatorname{deg}\left(f_{i_{1}, \ldots, i_{k}}^{c_{1}, \ldots, c_{k}}\right)+k\right\} .
$$


Доказательство. Рассмотрим в (11) подпространство $L=\left\{\mathbf{u} \in V_{m} \mid u_{i_{1}}=\ldots=u_{i_{k}}=0\right\}$ размерности $n-k$ и вектор $\mathbf{a}=\left(0, \ldots, 0, c_{1}, 0, \ldots, 0, c_{k}, 0, \ldots, 0\right)^{\top} \in V_{n}$, где $c_{j}$, $j=1, \ldots, k$, расположены на местах с номерами $i_{j}$. Ясно, что $\left.\mathrm{AI}\right|_{L \oplus \mathbf{a}} \leqslant \operatorname{deg}\left(f_{i_{1}, \ldots, i_{k}}^{c_{1}, \ldots, c_{k}}\right)$, так как в качестве аннигилятора функции $f$ (или $f \oplus 1$ в случае, если $f_{i_{1}, \ldots, i_{k}}^{c_{1}, \ldots, c_{k}}$ является константой 1) на $L \oplus \mathbf{a}$ можно взять отрицание подфункции $f_{i_{1}, \ldots, i_{k}}^{c_{1}, \ldots, c_{k}}$, если рассматривать его как функцию с $k$ несущественными переменными из $\mathscr{F}_{n}$.

Отсюда непосредственно следует справедливость утверждения.

Следствие 6. Для любой булевой функциии $f \in \mathscr{F}_{n}$

$$
\mathrm{la}(f) \geqslant \mathrm{AI}(f)-1 \text {. }
$$

Доказательство. Пусть $\mathrm{la}(f)=k$, тогда найдутся наборы $\mathbf{c} \in V_{k}, 0 \leqslant i_{1}<\ldots<i_{k} \leqslant n$, такие, что $\operatorname{deg}\left(f_{i_{1}, \ldots, i_{k}}^{c_{1}, \ldots, c_{k}}\right) \leqslant 1$. Отсюда с учетом (12) получаем, что $\operatorname{AI}(f) \leqslant 1+\operatorname{la}(f)$.

\section{6. Другие криптографические параметры}

Одним из криптографических параметров булевых функций является их алгебраическая степень. Как уже было замечено выше, с криптографической точки зрения высокая степень функций необходима для противостояния некоторым методам анализа.

В общем случае связи между алгебраической степенью функции и уровнем ее аффинности не наблюдается, что можно показать следующим утверждением.

Предложение 1. Для любых $n \geqslant 3,2 \leqslant d \leqslant n, 1 \leqslant k \leqslant n-2$ сущуествует функция $f_{n, d, k} \in \mathscr{F}_{n}$ такая, что $\operatorname{deg}(f)=d$, $\operatorname{la}(f)=k$.

Доказательство. Для случая $d=2$ можно рассмотреть функции вида

$$
f_{n, d, k}(\mathbf{x})=\bigoplus_{1 \leqslant i<j \leqslant k+1} x_{i} x_{j}
$$

а для $d \geqslant 2$ функции вида

$$
f_{n, d, k}(\mathbf{x})=\prod_{i=1}^{d} x_{i} \oplus \bigoplus_{1 \leqslant i<j \leqslant k+1} x_{i} x_{j} .
$$

Нетрудно видеть, что квадратичная часть этих функций обеспечивает необходимый уровень аффинности.

Рассмотрим так называемые лавинные характеристики булевых функций.

Функция $f \in \mathscr{F}_{n}$ удовлетворяет строгому лавинному критерию (SAC), если функция $\mathrm{D}_{\mathbf{u}} f=f(\mathbf{x} \oplus \mathbf{u}) \oplus f(\mathbf{x})$ является уравновешенной для любого $\mathbf{u} \in V_{n}$ такого, что $\mathrm{wt}(\mathbf{u})=1$. Функция $f \in \mathscr{F}_{n}$ удовлетворяет $\operatorname{SAC}(k)$, если подфункция $f_{i_{1}, \ldots, i_{k}}^{a_{1}, \ldots, a_{k}}$ при любых $1 \leqslant i_{1}<\ldots<i_{k} \leqslant n, \mathbf{a} \in V_{k}$ удовлетворяет SAC.

Заметим, что никакая аффинная функция $\ell_{\mathbf{a}, \varepsilon}$ не удовлетворяет $\mathrm{SAC}$, так как $\mathrm{D}_{\mathbf{u}} \ell_{\mathbf{a}, \varepsilon}$ есть константа для любого и. Отсюда непосредственно вытекает следующее очевидное утверждение.

Предложение 2. Для любой функиии $f \in \mathscr{F}_{n}$, удовлетворяющей $\mathrm{SAC}(t), \operatorname{la}(f) \geqslant t$. 
Следующие рассуждения показывают связь уровня аффинности функции с существованием у этой функции линейных структур.

Функция $f \in \mathscr{F}_{n}$ обладает линейной структурой, если в пространстве $V_{n}$ существует вектор $\mathbf{u} \neq \mathbf{0}$ такой, что

$$
\mathrm{D}_{\mathbf{u}} f(\mathbf{x})=f(\mathbf{x}) \oplus f(\mathbf{x} \oplus \mathbf{u})=\varepsilon_{\mathbf{u}}=c, \quad \varepsilon_{\mathbf{u}} \in F_{2},
$$

для любого $\mathbf{x} \in V_{n}$, здесь $c-$ постоянная.

Подпространство

$$
L_{f}=\left\{\mathbf{u} \in V_{n} \mid \mathrm{D}_{\mathbf{u}} f=c\right\},
$$

где $c$ - постоянная, называется подпространством линейности функции $f$.

Предложение 3. Пусть $f \in \mathscr{F}_{n}, \operatorname{deg}(f)>1$. Тогда

$$
\mathrm{la}(f) \leqslant n-\operatorname{dim} L_{f}-1 .
$$

Доказательство. Доказательство основано на том, что если $\operatorname{dim} L_{f}=r$, то функцию $f$ можно представить в виде (см. с. 107 в [4])

$$
f(\mathbf{x})=f(\mathbf{y}, \mathbf{z})=\ell(\mathbf{y}) \oplus g(\mathbf{z}),
$$

где $\ell(\mathbf{y}) \in \mathscr{A}_{r}, \mathbf{y} \in V_{r}, g(\mathbf{z}) \in \mathscr{F}_{n-r}-$ функция, не имеющая линейных структур, $\mathbf{z} \in V_{n-r}$. Таким образом, $\operatorname{la}(f)=\mathrm{la}(g) \leqslant n-r-1$.

В работах $[16,17]$ для исследования возможности линеаризации систем булевых уравнений введено понятие линеаризационного множества. Пусть $X=\left\{x_{1}, \ldots, x_{n}\right\}-$ множество переменных. Покрытием множества $X$ называется множество $B=\left\{B_{1}, \ldots, B_{k}\right\}$ такое, что $B_{i} \subseteq X, B_{i} \neq \varnothing, i=1, \ldots, k, \bigcup_{i=1}^{k} B_{i}=X$. Множество $B$ можно рассматривать как множество мономов некоторой булевой функции из $\mathscr{F}_{n}$.

Подмножество $L \subseteq X$ называется линеаризационным множеством покрытия $B$, если $|Y \backslash L| \leqslant 1$ для всех $Y \in B$. Линеаризационное множество наименьшей мощности называется кратчайшим. Обозначим мощность кратчайшего линеаризационного множества переменных функции $f$ через $l_{\min }(f)$.

Нетрудно понять, что, зафиксировав все переменные функции $f$ из некоторого соответствующего ей линеаризационного множества произвольным образом, мы получим аффинную функцию. Таким образом, $\operatorname{la}(f) \leqslant l_{\min }(f)$.

Приведем пример функции $f \in \mathscr{F}_{n}$, для которой $\mathrm{la}(f)<l_{\min }(f)$; такой является функция

$$
f(\mathbf{x})=x_{1} \varphi\left(x_{2}, \ldots, x_{n}\right) \oplus \varphi\left(x_{2}, \ldots, x_{n}\right), \quad \varphi \in \mathscr{F}_{n-1}, \quad \operatorname{deg}(\varphi)>1 .
$$

Действительно, поскольку $f_{1}^{1}=0$, очевидно, что $\operatorname{la}(f)=1$. В то же время $\operatorname{deg}\left(f_{i}^{0}\right)>1$ для любого $i, 1 \leqslant i \leqslant n$, то есть мощность кратчайшего линеаризационного множества больше 1 .

В заключение рассмотрим не сам уровень аффинности булевой функции, а понятие сильной $k$-аффинности и ее связь с индексом линейности.

Рассмотрим представление функций в виде линейного разветвления (см. [4]). Пусть $\rho: V_{n} \rightarrow V_{k}$. Будем говорить, что $f \in \mathscr{F}_{n}$ представима в виде линейного разветвления, если

$$
f(\mathbf{x})=\ell_{\rho(\mathbf{x})}(\mathbf{x}),
$$


где $\mathbf{x} \in V_{n}, \ell_{\mathbf{v}}(\mathbf{x})=\ell_{\mathbf{u}(\mathbf{v}), b(\mathbf{v})}(\mathbf{x})=\langle\mathbf{u}(\mathbf{v}), \mathbf{x}\rangle \oplus b(\mathbf{v}), \mathbf{u}(\mathbf{v}) \in V_{n}, b(\mathbf{v}) \in F_{2}$.

Минимальное натуральное $k$, для которого существует представление (13) функции $f$, называется индексом линейности этой функции и обозначается $\operatorname{ill}(f)$.

Предложение 4. Пусть $f \in \mathscr{F}_{n}$. Если функиия $f$ является сильно $k$-аффинной, то она представима в виде (13).

Доказательство. Пусть $f$ сильно $k$-аффинна, $k \geqslant 0$. Тогда существует такой набор $1 \leqslant i_{1}<\ldots<i_{k} \leqslant n$, что $f_{i_{1}, \ldots, i_{k}}^{a_{1}, \ldots, a_{k}}=\ell_{\mathbf{u}(\mathbf{a}), b(\mathbf{a})}$ для любого $\mathbf{a}=\left(a_{1}, \ldots, a_{k}\right)^{\top} \in V_{k}$. С учетом этого, раскладывая функцию $f$ по $k$ переменным, получаем, что

$$
\begin{aligned}
f(\mathbf{x}) & =\bigoplus_{\mathbf{a} \in V_{k}}\left(x_{i_{1}} \oplus a_{1} \oplus 1\right) \ldots\left(x_{i_{k}} \oplus a_{k} \oplus 1\right) f_{i_{1}, \ldots, i_{k}}^{a_{1}, \ldots, a_{k}}\left(\mathbf{x}^{\prime}\right) \\
& =\bigoplus_{\mathbf{a} \in V_{k}}\left(x_{i_{1}} \oplus a_{1} \oplus 1\right) \ldots\left(x_{i_{k}} \oplus a_{k} \oplus 1\right) \ell_{\mathbf{u}(\mathbf{a}), b(\mathbf{a})}\left(\mathbf{x}^{\prime}\right),
\end{aligned}
$$

где $\mathbf{x}^{\prime} \in V_{n-k}$.

Представление (14) легко приводится к представлению (13): достаточно положить $\rho\left(x_{1}, \ldots, x_{n}\right)=\left(x_{i_{1}}, \ldots, x_{i_{k}}\right)^{\top}$.

\section{7. Сложность задачи нахождения уровня аффинности}

Поскольку существует тесная связь между уровнем аффинности булевой функции и ее другими криптографическими параметрами, определенный интерес представляет задача определения уровня аффинности.

Если рассматривать представление функции как двоичный вектор ее значений (то есть, если $f \in \mathscr{F}_{n}$, то длина представления функции $f$ в этом случае есть $N=2^{n}$ ), то для произвольной булевой функции эта задача может быть решена тривиальным переборным алгоритмом за полиномиальное от длины входа время.

В этом разделе рассмотрим задачу отыскания уровня аффинности булевых функций в случае, когда число мономов в АНФ функции ограничено полиномиально от числа переменных. Мы покажем, что такая задача является $N P$-трудной.

Будем считать, что булева функция представляется мономами, входящими в ее АНФ (например, естественными номерами мономов). Введем множество

$$
\mathscr{F}_{c}(n)=\left\{f \in \mathscr{F}_{n}: \operatorname{len}(f) \leqslant c n\right\},
$$

где $c$ - постоянная и $\operatorname{len}(f)$ - число мономов в АНФ функции $f$. Заметим, что длина представления любой функции из $\mathscr{F}_{c}(n)$ полиномиально ограничена от $n$.

Докажем вспомогательное утверждение.

Предложение 5. Булева функция $f \in \mathscr{F}_{n}$, для которой $\operatorname{deg}(f) \leqslant 2$, является $k$-аффинной тогда и только тогда, когда $f$ является сильно $k$-аффинной.

Доказательство. Справедливость утверждения следует из того факта, что при любой фиксации какой-либо переменной $x_{i}$ соответствующий моном для квадратичной булевой функции переходит либо в константу, либо в линейный моном. Отсюда следует, что если функция $f_{i_{1}, \ldots, i_{k}}^{b_{1}, \ldots, b_{k}}$ является аффинной для какого-либо вектора $\mathbf{b}=\left(b_{1}, \ldots, b_{k}\right)^{\top} \in V_{k}$, то это верно и для любого вектора $\mathbf{c} \in V_{k}$. 
Рассмотрим задачу AFF, которая формулируется следующим образом: для заданной функции $f \in \mathscr{F}_{\mathrm{c}}(n)$ и заданного параметра $k$ требуется установить, является ли $k$ аффинной функция $f$.

Для доказательства $N P$-полноты этой задачи сведем к ней задачу о вершинном покрытии (ВП), которая состоит в том, чтобы определить, существует ли в заданном неориентированном графе $G=(V, E)$ без петель и кратных ребер вершинное покрытие мощности $q$ (см. [18]).

Теорема 8. Задача $A F F$ является $N P$-полной.

Доказательство. Сначала покажем, что AFF $\in N P$. Сертификатом (см. [18]) для этой задачи является набор переменных $x_{i_{1}}, \ldots, x_{i_{k}}$ и их значений $b_{1}, \ldots, b_{k}$. Проверку принадлежности соответствующей подфункции $f_{i_{1}, \ldots, i_{k}}^{b_{1}, \ldots, b_{k}}$ классу аффинных функций можно осуществить за полиномиальное от длины входа $c n$ время $(n-$ число переменных функции $f$ ).

Покажем теперь, что задача ВП полиномиально сводится к задаче AFF. Частным случаем задачи AFF является определение $k$-аффинности квадратичных булевых функций, то есть функций, для которых $\operatorname{deg}(f) \leqslant 2$ (все эти функции принадлежат $\mathscr{F}_{\mathrm{c}}$ ). В силу предложения 5 это эквивалентно определению, является ли соответствующая функция сильно $k$-аффинной. Рассмотрим произвольный граф $G=(V, E)$ без петель и кратных ребер и поставим в соответствие его вершинам переменные некоторой булевой функции. Также будем считать, что если $\left\{x_{i}, x_{j}\right\} \in E$, то в АНФ этой функции содержится моном $x_{i} x_{j}$. Таким образом, установлено соответствие между графами без петель и кратных ребер и квадратичными булевыми функциями. Нетрудно видеть, что, решая задачу AFF для квадратичных булевых функций, мы получаем решение задачи ВП для соответствующих графов: для вершинного покрытия достаточно взять набор вершин, соответствующий фиксируемым переменным. Далее, поскольку задача AFF является более общей, в силу описываемого в [19] метода сужения получаем, что AFF является $N P$-полной. Теорема 8 доказана.

Теорема 9. Задача нахождения уровня аффинности булевой функции из $\mathscr{F}_{\mathrm{c}}(n)$ является NP-трудной.

Доказательство. Очевидно, что задача AFF полиномиально сводится к задаче нахождения уровня аффинности: если $\mathrm{la}(f)>k$ ( $f$ и $k$ - параметры задачи AFF), то ответ задачи AFF - нет, в противном случае ответ - да. Отсюда в силу NP-полноты задачи AFF следует утверждение теоремы.

\section{Список литературы}

1. Логачев О. А., Сальников А. А., Ященко В. В., Корреляционная иммунность и реальная секретность. В сб.: Математика и безопасность информационных технологий. Материальк конференции в МГУ 23-24 октября 2003 г.. МЦНМО, Москва, 2004, с. 165-170.

2. Логачев О. А., Сальников А. А., Ященко В. В., Комбинирующие $k$-аффинные функции. В сб.: Математика и безопасность информачионных технологий. Материаль конференции в МГУ 23-24 октября 2003 г.. МЦНМО, Москва, 2004, с. 176-178.

3. Буряков М. Л., Логачев О. А., Об уровне аффинности булевых функций. Дискретная математика (2005) 17, №4, 98-107.

4. Логачев О. А., Сальников А. А., Ященко В. В., Булевы функции в теории кодирования и криптологии. МЦНМО, Москва, 2004. 
5. Таранников Ю. В., О корреляционно-иммунных и устойчивых булевых функциях. Математические вопросы кибернетики (2002) 11, 91-148.

6. Мак-Вильямс Ф. Дж., Слоэн Н. Дж. А., Теория кодов, исправляюших ошибки.. Связь, Москва, 1979.

7. Menezes A., van Oorschot P., Vanstone S., Handbook of applied cryptography. CRC, New York, 1997.

8. Siegenthaler T., Correlation-immunity of nonlinear combining functions for cryptographic applications. IEEE Trans. Inform. Theory (1984) 30, 776-780.

9. Courtois N., Pieprzyk J., Cryptanalysis of block ciphers with overdefined systems of equations.. Lect. Notes Comput. Sci. (2002) 2501, 267-287.

10. Courtois N., Patarin J., About the XL Algorithm over GF(2). Lect. Notes Comput. Sci. (2003) 2612, 141-157.

11. Courtois N., Meier W., Algebraic attacks on stream ciphers with linear feedback. Lect. Notes Comput. Sci. (2003) 2656, 345-359.

12. Meier W., Pasalic E., Carlet C., Algebraic attacks and decomposition of Boolean functions. Lect. Notes Comput. Sci. (2004) 3027, 474-491.

13. Matsui M., Linear cryptanalysis method for DES cipher. Lect. Notes Comput. Sci. (1993) 765 , 386-397.

14. Rothaus O., On bent function. J. Combin. Theory (1976) A20, 300-305.

15. Dalai D. K., Gupta K. C., Maitra S., Results on algebraic immunity for cryptographically significant Boolean functions. Lect. Notes Comput. Sci. (2004) 3348, 92-106.

16. Тимошевская Н. Е., О линеаризационных множествах. В сб.: Проблемы теоретической кибернетики. Изд-во механико-математического факультета МГУ, Москва, 2005, с. 154.

17. Тимошевская Н. Е., Задача о кратчайшем линеаризационном множестве. Вестник Томского гос. унив. (2005) 14, 79-83.

18. Алексеев В. Б., Введение в теорию сложности алгоритмов. Изд-во факультета ВМиК МГУ, Москва, 2002.

19. Гэри М., Джонсон Д., Вычислительные машины и труднорешаемые задачи. Мир, Москва, 1982.

Статья поступила 11.12.2007. 